\title{
COMMENTARY
}

\section{Anthroposphere, A New Physical Dimension of the Ecosystem}

\author{
Ji-Dong Gu \\ School of Biological Sciences, The University of Hong Kong, Pokfulam Road, Hong Kong SAR, China
}

\begin{abstract}
Ecosystem is having a new physical dimension as evidenced by the increasing contribution from man-made plastics synthesized and consumed by our society. Anthroposphere, a brand new constituent, shall be added into the existing lithosphere, hydrosphere, atmosphere and biosphere as a whole for the ecosystem. This new class of anthropogenically-produced materials is xenobiotic and has its own physical form persistent and building up in the ecosystem. Its occurrence in different ecosystems will increase continuously over time. Its hazards and potentially effects in different ecosystems shall be evaluated with a new framework to advance a better understanding of its long-term impact and the mechanisms involved in the changing world. The impacts from these plastics shall be much more than its non-degradability and the potential hazards as observed and a better understanding based on pure science is required. A new opportunity is ahead of us for a collective and immediate action to assess the new dimension of the anthroposphere in the ecosystems to advance the new knowledge on this research subject.
\end{abstract}

Keywords: Anthrome, anthropocene, ecotoxicology, metals, persistent organic pollutants

*Correspondence to: Ji-Dong Gu, School of Biological Sciences, The University of Hong Kong, Pokfulam Road, Hong Kong SAR, China;

E-mail: jdgu@hku.hk

Received: January 8, 2020; Accepted: February 13, 2020; Published Online: February 14, 2020

Citation: Ji-Dong Gu, 2020. Anthroposphere, A New Physical Dimension of the Ecosystem. Applied Environmental Biotechnology, 5(1):34-36. http://doi.org/10.26789/AEB.2020.01.001

Copyright: Anthroposphere, A New Physical Dimension of the Ecosystem.@ 2020 Ji-Dong Gu. This is an Open Access article distributed under the terms of the Creative Commons Attribution-Noncommercial 4.0 International License, permitting all non-commercial use, distribution, and reproduction in any medium, provided the original work is properly cited and acknowledged.

Human and their activities have changed the biochemical functional processes in the ecosystem undoubtedly. Global warming caused is one example by high concentration and building up of $\mathrm{CO}_{2}$ from combustion into the atmosphere on earth and nitrogen fertilizer is another one. At the same time, different parts of the natural ecosystem have experienced a significant alteration through the manufacturing and release of large quantities of xenobiotics by industries for various purposes, including protection for crops and eradication of harmful insects (Schwarzenbach et al., 2006). This class of chemicals possesses unique chemical structures that have never been part of the ecosystem, and their existence has been expanded further into medicine and household materials on a daily basis in the modern lifestyle. These chemicals are extremely resistant to (bio)degradation, especially by microorganisms of the natural habitats to result in further building up or accumulation of them in the environment, namely lithosphere, hydrosphere and biosphere (Gu, 2018a). Greater efforts have been put into the research on the elimination and also assessment on the fate of them over the past half a century (Stotzky, 1986; Stumm and Morgan, 1996; Alexander, 1999; Schwarzenbach et al., 2006; Gu, 2019a), and no good effective measures have been identified or discovered after so many years of attempts.

In addition to the persistence nature in the ecosystem, they are transported through air movement from the areas of production and use to those without any production or utilization. At any specific location, they are also partitioned in the ecosystem, namely lithosphere, hydrosphere, atmosphere and biosphere (Schwarzenbach et al., 2006). Because of the persistence and bioaccumulation of them, they are widely present in the ecosystem, and the toxicity of them is a key topic of our concern to human and ecosystem.

The large number of xenobiotics manufactured and produced has been a fact of life in the industrialized nations and developing countries. Because of this trend, a wider spread and more intensified accumulation of them is evident in the natural ecosystem (Ackerman, 2014) and some confirmed negative impacts to human and animals are also evident (Carson, 1962). Apart from these chemicals, another class of materials has been recognized for their ubiquity in the ecosystem, soot carbon from combustion of petroleum and also natural fire or incomplete incineration and burning. The soot carbon is a product of natural product and they are very fine carbon-based materials in the sediment mainly. It has very unique properties in terms of sorption and accumulation for hydrophobic chemicals. More recently, a new class of chemicals in the ecosystem attracts the attention of the public because of their high volume of demand and consumption, plastics. This class of materials is the fast growing, and increasingly manufactured and consumed materials from daily use to infrastructure and aviation, space and military ( $\mathrm{Gu}, 2003)$. It is impossible to image a day without them now. They have been wide found in oceans, sediments, atmosphere and biota. 


\section{A New Physical Dimension}

Plastics are diverse in their chemical structures and also applications, but they are fundamentally different from other conventional chemicals in that the formers are polymerization products with various chain lengths to obtain the physical properties in application. They have distinctive physical forms as sheets of plastic in packaging and mulching to bottling and structural applications. In this way, plastics are different from other chemical toxicants in that the formers are physically identifiable easily from the ecosystem and they are not soluble in water. After disintegration due to physical, chemical and possibly biological means, the fragments, also called micro-plastics, are still physical intact particles in the ecosystems. By doing so, plastics, large or small, are different from the conventional pollutants, e.g., DDT, Lindane, PCB and PAHs, which can penetrate into micro-pores of the sediments and sorbed onto clay minerals and into humus matter, so they can become part of the inorganic, organic or complexes in the lithosphere.

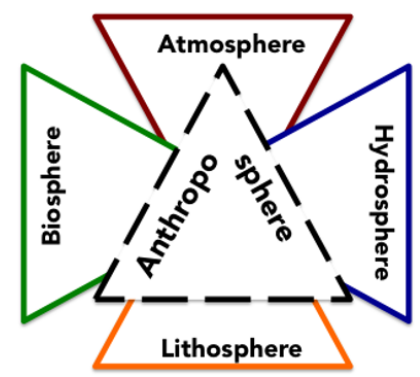

Figure 1. A new proposed scheme on the composition of the ecosystem with the 5 spheres including the newly proposed anthroposphere

This physical distinctiveness of plastics makes them interact with the environmental differently in many ways. Plastics are produced from natural petroleum, but their chemical structures are never parts of the ecosystem before, now or afterward. The highly polymerization nature of plastics in their molecular structures gives them the unique characteristics for application as durable materials for service and strength. This modification from natural petroleum has made them unique and very different from any materials naturally occurring in the ecosystems. They are persistent, distinctive, and, much more, foreign to the natural ecosystems. Because of these, they are becoming permanent constituents of the ecosystem now. Due to the persistent properties, plastics and micro-plastics are new physical materials released into the ecosystem and become a new physical identity of the ecosystem and a new dimension introduced into the environment with increasing presence and influence, which attract the attention of general public and scientists. Based on these, this new dimension of the ecosystem shall be recognized when ecosystem and function are assessed now and in the future. Anthroposphere is a new addition to the ecosystem constituents (Figure 1).

The physical world of ecosystem is known consisted of lithosphere, hydrosphere, atmosphere and biosphere in introductory ecology, but now a new anthroposphere has emerged. This anthroposphere must be recognized from now on because their presence has been for a while and their impacts are becoming known, but largely ignored by limited information available. Because of the increasing consumption and manufacturing of them, this constituent in the ecosystem will increase further to their share of the whole ecosystem mass. The concentration of plastics and micro-plastics in the environments, e.g., water, sediment, air or organisms, can be measured, but their concentration by mass is different from the conventional chemicals because plastics are not miscible in aqueous phase, especially water, so their interactions with the physical environmental constituents are different.

\section{Concentration and Impact Assessment}

Plastics and micro-plastics can be extracted from the different parts of the ecosystems, but a uniform concentration of them is almost impossible due to their physical forms and diverse chemistry in structures and composition. The concentration of any plastics used in an assay is a highly questionable one because they are physically distinguished from aqueous phase and do not form homogenous solution in water. When this is the fact, the concentration of microplastics does not exist in any solvent that does not dissolve the plastics. Given the fact that some very selective solvents can dissolve plastics, but such homogenous system does not allow conducting toxicity tests which depend on living organism or microorganisms as the testing samples. If the concentration cannot be established with general plastics, the data on their toxicity is misleading. Some of the study conducted simply by putting some amount of plastic micro-beads with the exposed organisms in water to observe any effects on the organisms, which cannot be regarded as a good toxicological test. This practice must be disqualified to safeguard the science and development.

\section{Future Perspectives}

Plastics as a new dimension of the ecosystem, be the anthroposphere, are becoming increasing recognized by the public. Being a physically distinguished from other constituents of the earth ecosystem, plastics shall be recognized as anthroposphere to advance further conceptual framework of research and study from now onward. Many questions are emerging to the appearance of this new dimension of the natural world on their effect on the natural cycling of nutrients, their influences on the community of indigenous microorganisms and biological processes, the fate of them in the long-term after disposal into ecosystem. Are they actually biodegraable and to what extent? It is clear that we know many pollutants and their fate in the ecosystem, but we know nothing about this class of new materials.

\section{Conflict of Interest}

Author declares that there is no conflict of interest in this research. 


\section{Ethical approval}

This article does not contain any studies with human participants or animals performed by the author involved.

\section{References}

Ackerman, D., 2014. The Human Age: The World Shaped by Us. W.W. Norton, New York. 352 pp.

Alexander, M., 1999. Biodegradation and Bioremediation ( $2^{\text {nd }}$ ed.). Academic Press, San Diego, California.

Carson, R., 1962. Silent Spring. Penguin Books, Ltd, London, UK, pp. 336

Gu, J.-D., 2016. Biodegradation testing: so many tests but very little new innovation. Applied Environmental Biotechnology, 1(1): 92-95.

https://doi.org/10.18063/AEB.2016.01.007

Gu, J.-D., 2018a. The endocrine-disrupting plasticizers will stay with us for a long time. Applied Environmental Biotechnology 3 (1): 61-64. https://doi.org/10.26789/AEB.2018.01.008

Gu, J.-D., 2018b. Bioremediation of toxic metals and metalloids for cleaning up from soils and sediments. Applied Environmental Biotechnology 3 (2): 48-51. https://doi.org/10.26789/AEB.2018.02.006
Gu, J.-D., 2019a. Microbial ecotoxicology as an emerging research subject. Applied Environmental Biotechnology 4 (1): $1-4$. https://doi.org/10.26789/AEB.2019.01.001

Gu, J.-D., 2019b. On applied toxicology. Applied Environmental Biotechnology 4 (2): 1-4. https://doi.org/10.26789/AEB.2019.02.001

Schwarzenbach, R.P., B.I. Escher, K. Fenner, T.B. Hofstetter, C.A Jonson, U. von Gunten and B. Wehrli, 2006. The challenge of micropollutants in aquatic systems. Science 313: 10721077.

https://doi.org/10.1126/science.1127291

Stotzky, G., 1986. Influence of soil mineral colloids on metabolic processes, growth, adhesion, and ecology of microbes and viruses. Pp. 305-428. In P.M. Huang and M. Schnitzer, eds. Interactions of Soil Minerals with natural Organics and Microbes. SSSA Special Publication No. 17. Soil Science Society of America, Inc., Madison, WI https://doi.org/10.2136/sssaspecpub17.c10

Stumm, W. and Morgan, J.J., 1996. Aquatic chemistry: chemical equilibria and rates in natural waters. ( $3^{\text {rd }}$ ed.), Wiley, New York. pp. 1022. 